\title{
Adaptive Neural Network Fuzzy Inference System for HFC Processes
}

\author{
Victor Vladareanu ${ }^{1}$, Luige Vladareanu ${ }^{1}$, Radu Ioan Munteanu ${ }^{2}$, Lucian Capitanu ${ }^{1}$, \\ Emilian M. Dobrescu, Mihai Rădulescu ${ }^{1}$, Vlad Alexandru Grosu ${ }^{3}$ \\ ${ }^{1}$ Robotics and Mechatronics Dept., Institute of Solid Mechanics of the Romanian Academy, \\ Bucharest, Romania, \\ ${ }^{2}$ Technical University of Cluj-Napoca \\ ${ }^{3}$ Faculty of Electronics, Telecommunications and Information Technology, University Politehnica of Bucharest, Bucharest, Romania
}

\begin{abstract}
The paper presents the design and implementation of a fuzzy inference system (FIS) trained with adaptive neural networks for the generation of specification references in high frequency current (HFC) hardening processes. The specification references are then further used for the control of the process in obtaining the desired outcomes in terms of material hardening and resistance. The FIS is trained using data obtained from experimentation on an industrial HFC device. The trained FIS is then compared to a manually tuned FIS, resulting from expert and operator designs. The results led to the development of intelligent control interfaces in real time through the ANFIS method.
\end{abstract}

intelligent control, real time control systems, hardening process, materials, highfrequency currents.

\section{Corresponding Author:}

Luige Vladareanu,

Romanian Academy, Institute of Solid Mechanics,

15 Constantin Mille street, 010141 Bucharest, Romania.

Email: luige.vladareanu@vipro.edu.ro

\section{INTRODUCTION}

The control system for HFC process robots (thermal treatments by high-frequency hardening) is based on the magnetic induction phenomenon and the film effect, according to which the piece to be hardened is introduced in a magnetic field where an electric current is induced, which is distributed only in the peripheral layers of the work piece, reaching the temperature level required by the thermal treatment conditions in a very short time in these superficial layers. The superficial layer, reaching the temperature in a very short time, is hardened, while there are no structural changes in the core and therefore no change of the initial properties. The surface hardening offers a distribution of internal stresses on the section, which leads at the increasing of fatigue resistance, at minimal deformations (because the core remains rigid). Consequently, the productivity increases as a result of heating in a relatively short amount of time. The process of high-frequency current quenching is especially popular in serial production, offering besides high productivity and superior quality of the parts being treated. The process ensures a rigorous reproducibility of heating conditions for the entire batch of pieces and is suited in the process mechanization and automation (Rapoport et all, 2006; Yogesh et all, 2002; Rudnev et all, 2002).

The Reference Generation Module is used to specify the desired references for high frequency quenching variables designed as output variables, depending on the setting of the two input variables. These are the 
movement speed of the piece, the gap between the piece and the inductor and the power emitted by the inductor during quenching. In most applications, the obvious relationship is desirable to specify that the hardness and strength of the superficial layer depend on speed, interstitial and power. In this context, the relationship of dependence is reversed because is desirable to specify some references for the variables controlled in the HFC process (speed, gap and power), depending on the desired results for hardness and strength (Dumitrache et al., 2017, Moisescu et al., 2016).

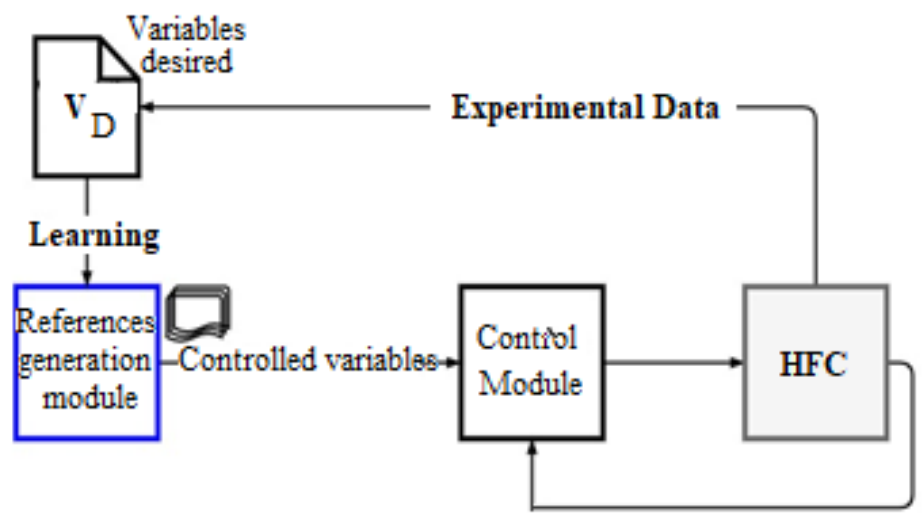

Figure 1. Reference Generation Module

On the basis of the conclusions reached in the previous activities, referring to the modelling and control of the high frequency hardening using the VIP platform, in the previous stage was designed a fuzzy decision system for setting the input parameters of the HFC equipment. This system is based on the conclusions reached in the previous stage, where the expertise of the academic and industrial specialists has been sampled and systematized (Vladareanu L. et all, 2009; Barz C. et al. 2016a, 2016b, Vladareanu V. et all 2015).

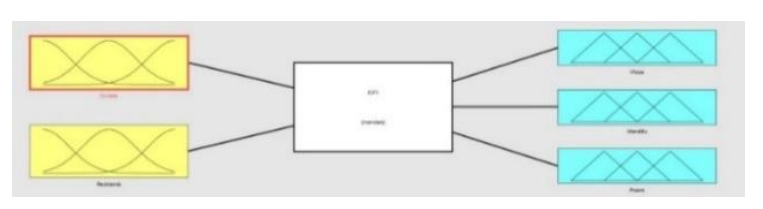

Figure 2. Fuzzy Inference System for HFC Control

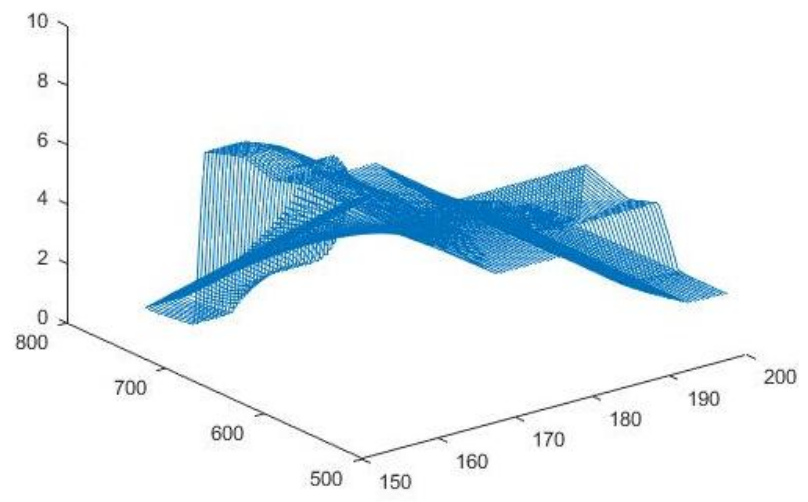

Figure 3. Learned FIS

The HFC fuzzy decision system serves to identify references for HFC control variables in order to achieve the desired behaviour. This model eliminates the need to query the human experts, being a completely automated process. Learning is done using the ANFIS method - a fuzzy inference system optimized using the adaptive neural networks - that requires the existence of a data field to be run obtained from practical experiments. As a result of validation and practical demonstration of this model, a fuzzy decision system could be set up to specify the references of HFC equipment control system directly from the present experimental data loaded into the platform. The main advantage is the possibility of generating an optimized fuzzy inference system for situations where the experience and expert expertise is absent or divergent (Boscoianu M. et all, 2018, Vladareanu L. et all, 2017; Wang HB et all, 2017;). A diagram of the fuzzy decision-making process as a black-box model is presented in Fig. 2. The two inputs and the three outputs explained above are shown as fuzzy variables. 


\section{HFC ANFIS}

ANFIS is trained using a number of examples obtained in practical HFC equipment tests. Figure 3 provides an example of learning space for the speed variable, consisting in all examples used to train this specific algorithm. One of the ANFIS advantages is that for points situated between the existing examples, the result will be approximated using a combination of rules triggered by the closest inputs. Once trained, the resulting fuzzy inference system can be used in any simulation as a reference table to specify the reference values of the controlled quantities. The membership functions on the basis of which the fuzzification process is made are also different (web-1).

Manually tuned FISs mainly contain trapezoidal and triangular membership functions (example in Fig. 4), while the ANFIS process is typically run using Gauss membership functions, such as the distribution presented in Fig. 5.

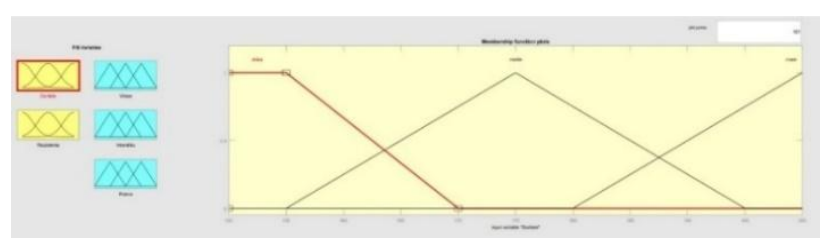

Figure 4. Manually tuned FIS with trapezoidal MFs

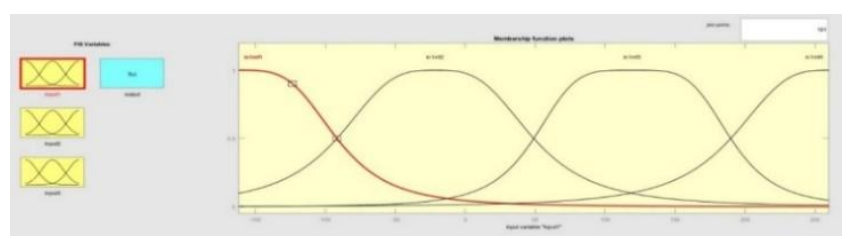

Figure 5. ANFIS with Gaussian MFs

Also, the trained fuzzy inference system is not of Mamdani type, which is most common. Mamdani systems use common fuzzy membership functions for the output variable, similar to those used for the input variable. This distribution is inefficient in the case of learning processes, being very computationally intensive. Instead of the Mamdani inference systems, the Sugeno type is used, where the distribution of the output variable is composed of constant (Sugeno type 0) or linear (Sugeno type 1) functions.

To adapt to the learning process, the decision system is not a single inference system (FIS) integrated with 2 inputs and 3 outputs, as in the previous case, but 3 inference systems (FIS) with 2 inputs and one output, constituted in a general system that will call one of the inference systems, depending on the required variable. The boundaries of the universe of discourse of each variable are described in Table 1.

\begin{tabular}{l|lll} 
Variable & Inferior limit & Upper limit & Unit \\
\hline Hardness & 150 & 200 & $\mathrm{HV}$ \\
Resistance & 520 & 730 & $\mathrm{~N} / \mathrm{mm} 2$ \\
Speed (rate) & 0 & 10 & $\mathrm{~mm} / \mathrm{s}$ \\
Gap (interstice) & 0 & 10 & $\mathrm{Mm}$ \\
Power & 0 & 50 & $\mathrm{~kW}$
\end{tabular}

Table 1. Variable boundaries

The ANFIS algorithm will therefore run for learning of each output variable (velocity, gap, power) separately on a data structure containing 3 columns, the first two being the input variables (hardness and strength), and the third the learning output variable as Data $=$ [DR Target $]$.

The optimization consists in the successive improvement of the inference system that models the interaction between the independent input variables and respectively the dependent output variables in the context of the available data set. This implies the existence of an initial inference system, which is obtained by automatic generation based on the data set. The algorithm implicitly calls a generating function, but this behaviour can be changed by the user. The fuzzy inference system generated by genfis 1 always requires a subsequent optimization by ANFIS, while the system generated by genfis 2 can have a sufficient behaviour for less complex cases. The experimental model uses both generating options for the initial fuzzy inference system. 


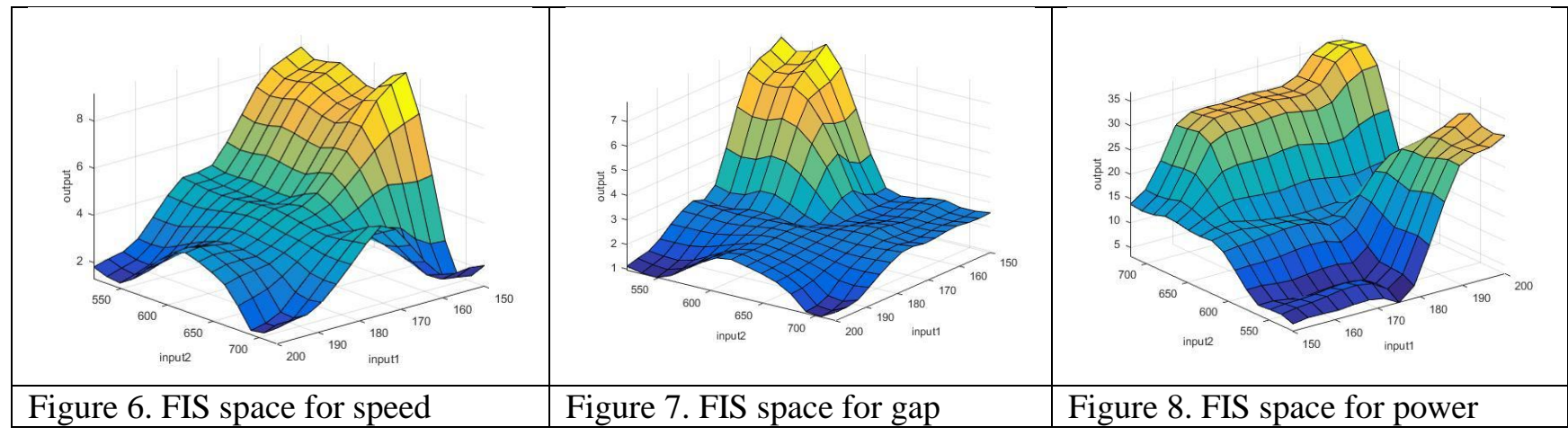

After running the optimization algorithm, three inference systems are automatically obtained and tested, one for each output variable shown in figures 6-8. Each figure shows the inference space for an output variable relative to the input variables. It can be seen from Figures 6-8 the modelling for each variable of a strongly non-linear behaviour, based on optimized inference rules. Using the specification of the fuzzy rules and the inference space it can deduce the action of the HFC fuzzy decision system in the case of different input data sets. This value will serve as a reference for each of the three HFC process sizes controlled by us, namely the output variables of the current application: speed, interstitial, and power.

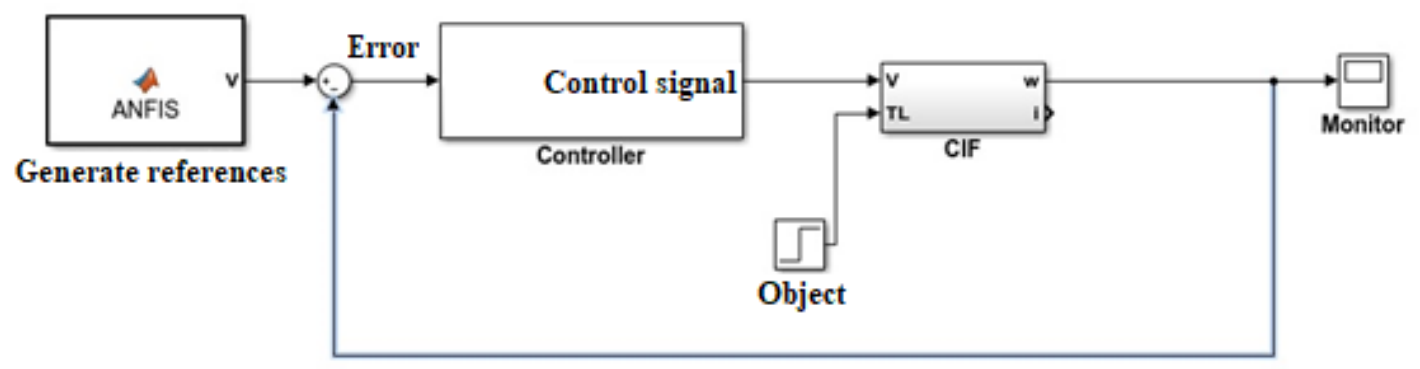

Figure 9. FIS reference generation module

The elaboration of the experimental module of the fuzzy-HFC decision system is one of the reference specification methods for the controlled HFC intelligent process sizes. The scheme of this concept is presented in Figure 9.

\section{EXPERIMENTAL RESULTS}

The application is tested varying their input sizes between their limits. In the case of the input variables, the limits are: $d=150 \ldots 200, r=520 \ldots 720$. Also, the output variables have their own limits modelled in the system frame which is to be tested. These are: $v=0 \ldots 10, i=0 \ldots 10, p=0 \ldots 50$.

The experimenting of the ANFIS HFC functional model is done in parallel with the HFC fuzzy inference system obtained in the previous stage with the experts consensus. For this purpose, a random data set for input values is generated, which is evaluated using the HFC fuzzy inference system, resulting a data field for the output variables. It is learned through the ANFIS process, so resulting in an alternative fuzzy inference system.

Figure 10 shows a diagram of the test method for the two inferential processes. The HFC fuzzy system has three outputs, which will be demultiplexed to obtain each variable, while ANFIS HFC requires the learning of three separate inference systems, one for each output variable. Differences between the two systems are viewed, individually per variable, and gathered in absolute value to obtain a quantification norm. 


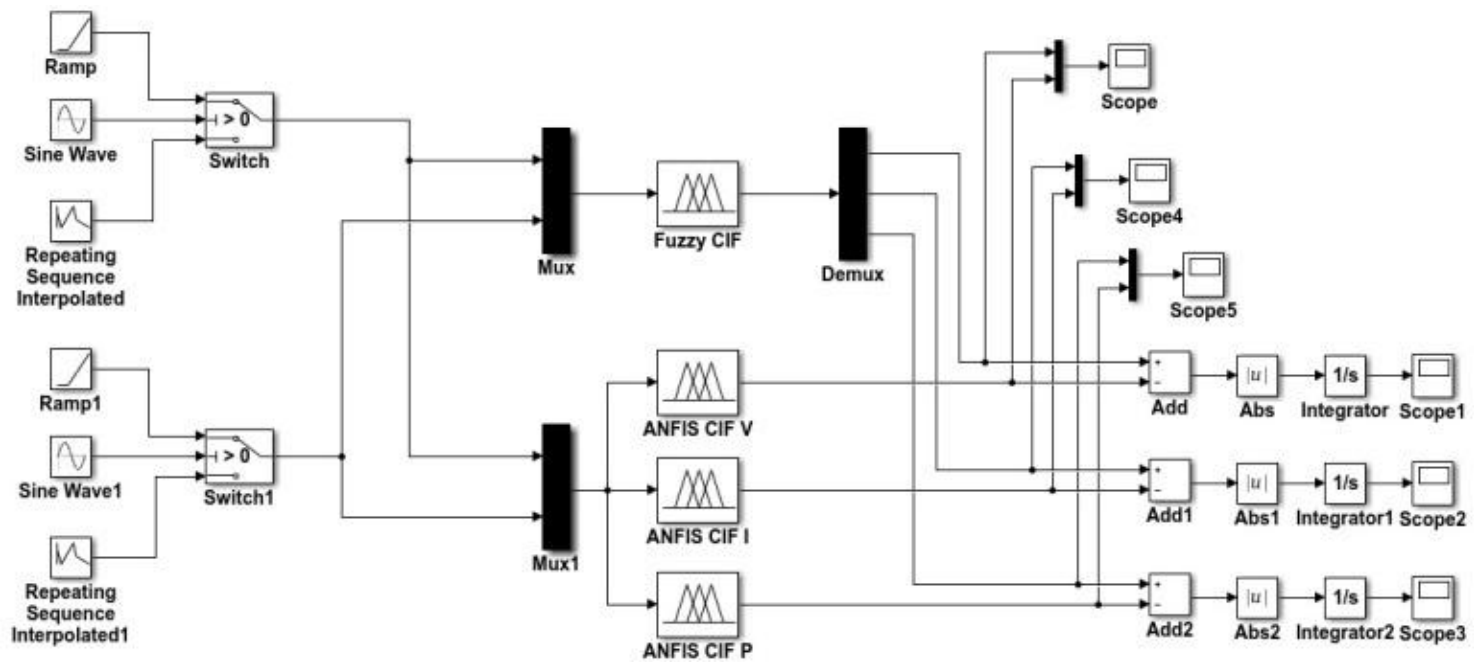

Figure 10. Diagram of testing

To experience the ANFIS HFC module and explain the differences towards the HFC fuzzy module, three sets of input data were generated and used for both systems. The test kits have 10 points per input variable, resulting in 100 test points formed from all possible combinations of them. These sets were developed according to laws similar to those used for the initial experimentation of the HFC fuzzy module and chosen to pass through the entire input space of the inference system to observe the responses.
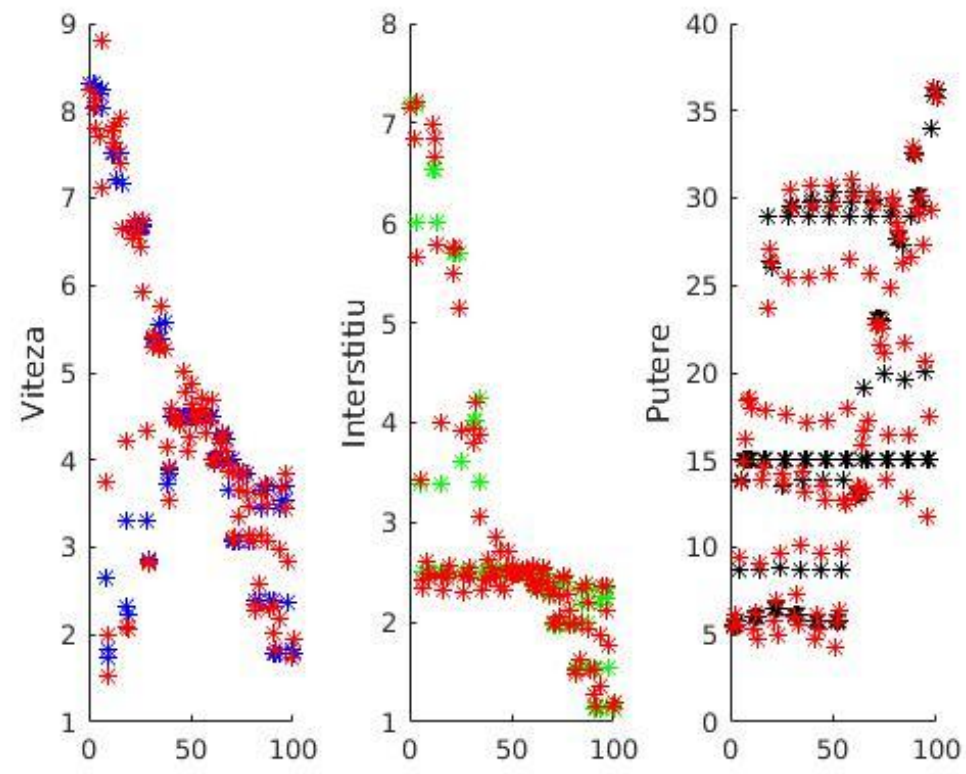

Figure 11. Results of testing ANFIS and manually tuned FIS

The results of the test are shown in Figure 11. The output values of the HFC fuzzy inference system are marked with the colour specific to the output variable, speed, gap (interstice) or power. The values proposed by the ANFIS HFC inference system are marked in red to highlight the contrast between the two systems.

It can be noticed that although they do not have the same behaviour, the two inference systems offer similar values for the output variables throughout the workspace run. This conclusion is also supported by the values of the absolute mean difference per variable, namely: 
Experiment

Speed

Gap (interstice)

Power
Absolute average difference

0.2116

0.1119

1.2865

Table 2. Experiment results

Following the tests, it can be concluded that the two inferential systems successfully model the same application, providing HFC control references for three output variables, based on the desired specifications, modelled by the input variables. The HFC fuzzy system is based on accumulated human experience, while the ANFIS HFC system is learned from the experimental data, obtained and generated.

\section{RESULTS AND CONCLUSIONS}

HFC Hardening is an important part of industrial processing in construction applications. Its automation requires a complex control system, an important part of which is the reference generation module. An earlier version based on experimental data and expert queries was improved based on the automated learning of a fuzzy inference system (FIS) using adaptive neural networks.

The advantages of the second system consist in the repeatability and flexibility of the process, as well as in the non-linear behaviour of output data generated within the same class, due to the way in which the inference system was obtained. These features have also been seen and described in this experimental work, which will be further used to demonstrate the utility and functionality of the model and for implementation in the VIP smart platform, which controls the HFC process.

The results obtained outline the development of the ICI modules using advanced control strategies adapted to the induction hardening process, applying ICT techniques with fast processing and real-time communications, which led to improvement of the material's mechanical characteristics.

\section{ACKNOWLEDGEMENTS}

This work was supported by a grant of the Romanian Ministry of Research and Innovation (MCI), CCCDIUEFISCDI, MULTIMOND2 project number PN-III-P1-1.2-PCCDI2017-0637/33PCCDI/01.03.2018, and by KEYTHROB project, within PNCDI III, MCI and MFE by Competitiveness Operational Programme (COP) / "Programul Operațional Competitivitate" (POC), TOP MetEco AMBIENT project, ID P_39_383, contract107/09.09.2016, and by the European Commission Marie Skłodowska-Curie SMOOTH project, Smart Robots for Fire-Fighting, H2020-MSCA-RISE-2016-734875. The authors gratefully acknowledge the support of the Robotics and Mechatronics Department, Insitute of Solid Mechanics of the Romanian Academy.

\section{References}

[1] Barz C., Ilia M., Ilut T., Pop-Vadean A, Pop P.P., Dragan F.,(2016a), Weintek interfaces for controlling the position of a robotic arm, IOP Conf. Ser.: Mater. Sci. Eng. 144 012018,

[2] Barz C., Todea C. et al., (2016b), Intelligent traffic control system using PLC, IOP Conf. Ser.: Mater. Sci. Eng. 144 012017, https://iopscience.iop.org/article/10.1088/1757-899X/144/1/012017 
[3] Boscoianu, M., Prelipcean, G., Lupan, M., (2018), Innovation enterprise as a vehicle for sustainable developmnet- A general framework for the design of typical strategies based on enterprise systems engineering, dynamic capabilities, and option pricing, Journal of Cleaner Production vol 172, p3498-3507;

[4] Dumitrache, I., Sacala, I. S., Moisescu, M. A., \& Caramihai, S. I. (2017). A conceptual framework for modeling and design of Cyber-Physical Systems. Studies in Informatics and Control, 26(3), 325-334.

[5] Moisescu, M. A., \& Sacala, I. S. (2016). Towards the development of interoperable sensing systems for the future enterprise. Journal of Intelligent Manufacturing, 27(1), 33-54.

[6] Rapoport, Edgar; Pleshivtseva, Yulia (2006), Optimal Control of Induction Heating Processes, CRC Press, Series: Mechanical Engineering, 349 pp., ISBN 9780849337543;

[7] Rudnev, Valery; Loveless, Don; Cook, Raymond; Black, Micah (2002), Handbook of Induction Heating, CRC Press, ISBN 0-8247-0848-2;

[8] Vladareanu L., Velea L.M., Munteanu R.I, Curaj A., Cononovici S., Sireteanu T., Capitanu L., Munteanu M.S., (2009), Real time control method and device for robots in virtual projection, patent EPO-09464001, 18.05.2009, EP2105263. Patent OSIM 123527/30.04.2013;

[9] Vlădăreanu L., Vlădăreanu V., Șchiopu P., (2012), Hybrid Force-Position Dynamic Control of the Robots Using Fuzzy Applications, 3-rd Edition of the IEEE/IACSIT International conference on Biomechanics, Neurorehabilitation, Mechanical Engineering, Manufacturing Systems, Robotics and Aerospace, ICMERA2012, Bucharest, pp.8, Invited Paper;

[10] Vladareanu V., Ioan Dumitrache, Luige Vladareanu, Ioan Stefan Sacala, Gabriela Tonţ, Mihnea Alexandru Moisescu, (2015), "Versatile Intelligent Portable Robot Platform applied to dynamic control of the walking robots", Studies in Informatics and Control 24(4):409-418 • December 2015, ISSN: 1220 - 1766;

[11] Wang H. B.; Yan H.; Feng Y. F., et al., Design and kinematics simulation of a fingertip-guided rehabilitation robot, (2017) IEEE International Conference on Real-time Computing and Robotics (RCAR), Okinawa, Japan, https://ieeexplore.ieee.org/document/8311874

[12] Wang H.B.; Liu Z.; He X.; Niu J.; Lan Z., (2016),Motor controller design of patient transfer apparatus, 2016 IEEE International Conference on Information and Automation (ICIA), https://ieeexplore.ieee.org/document/7831943

[13] Yogesh Jaluria, Computational Heat Transfer, November 21, 2002 by CRC Press, Series: Series in Computational and Physical Processes in Mechanics and Thermal Sciences, 560 pp, ISBN 9781560324775

[14] Web-1: http://www.cs.nthu.edu.tw/ jang/anfisfaq.htm, retrieved Dec 2018; 